COMMUNICATIONS IN

ANALYSIS AND GEOMETRY

Volume 12, Number 1, 165-182, 2004

\title{
Brownian Motion on a Submanifold
}

\author{
DANIEL W. STROOCK
}

\begin{abstract}
Given a submanifold $M$ of a Riemannian manifold $N$, we give two different constructions of Brownian motion on $M$ : one by "projection" onto $M$ of the Brownian motion on $N$ and the other by a more intrinsic approach. The two procedures lead to very different ways in which vectors are transported along Brownian paths.
\end{abstract}

\section{Introduction.}

Throughout this note $N$ will denote a complete, connected $n$-dimensional Riemannian manifold and $M$ will be a closed $m$-dimensional, imbedded submanifold of $N$ which is given the Riemannian structure which it inherits from $N$. In addition, we will be using $\nabla^{N}$ to denote the Levi-Civita on $N$, and $\nabla^{M}$ to denote the inherited Levi-Civita on $M$. Finally, given a piecewise smooth path $p:[0, t] \longrightarrow N$, we will use $\mathcal{T}_{p}^{N} \in \operatorname{Hom}\left(T_{p(0)} N ; T_{p(t)}\right)$ to denote parallel transport along $p$. Similarly, if $p$ takes its values in $M$, then $\mathcal{T}_{p}^{M} \in \operatorname{Hom}\left(T_{p(0)} M ; T_{p(t)} M\right)$ will be parallel transport along $p$ as a path in $M$.

Our goal is to examine various relations between the Brownian motion on $N$ and the Brownian motion on $M$. This sort of analysis was carried out in Chapters 4 and 5 of [3] when $N=\mathbb{R}^{n}$. However, even in that case, the analysis given there is less complete than the one given here.

\section{The Shape Operator.}

Given $x \in M$, define the shape operator $\mathcal{S}_{x} \in \operatorname{Hom}\left(T_{x} M ; \operatorname{Hom}\left(T_{x} N ; T_{x} N\right)\right)$ so that if $X_{x} \in T_{x} M$, then

$$
\mathcal{S}_{x}\left(X_{x}\right)=\left.\frac{d}{d t}\left(\mathcal{T}_{p \uparrow[0, t]}^{N}\right)^{-1} \circ \Pi_{p(t)} \circ \mathcal{T}_{p \uparrow[0, t]}^{N} X_{x}\right|_{t=0},
$$

\footnotetext{
${ }^{1}$ Research contained in this article was partially supported by NSF grant DMS \#6890947.
} 
where $p \in C^{1}([0, \infty) ; M)$ with $p(0)=x$ and $\dot{p}(0)=X_{x}$. To see that $\mathcal{S}_{x}\left(X_{x}\right)$ is well-defined (i.e., independent of the choice of $p$ ), observe that if $Y \in T N$ is any vector field on $N$, then

$$
\begin{aligned}
\nabla_{X_{x}}^{N}(\Pi Y) & =\left.\frac{d}{d t}\left(\left(\mathcal{T}_{p\lceil[0, t]}\right)^{-1} \circ \Pi_{p(t)} \circ \mathcal{T}_{p\lceil[0, t]}\right)\left(\mathcal{T}_{p \uparrow[0, t]}\right)^{-1} Y_{p(t)}\right|_{t=0} \\
& =\mathcal{S}_{x}\left(X_{x}\right) Y_{x}+\Pi_{x} \nabla_{X_{x}}^{N} Y,
\end{aligned}
$$

and so

$$
\mathcal{S}_{x}\left(X_{x}\right) Y_{x}=\nabla_{X_{x}}^{N}(\Pi Y)-\Pi_{x} \nabla_{X_{x}}^{N} Y
$$

In particular,

$$
\begin{aligned}
Y \uparrow M \in T M \Longrightarrow \mathcal{S}_{x}\left(X_{x}\right) Y_{x}=\Pi_{x}^{\perp} \nabla_{X_{x}}^{N} Y & \\
& =\nabla_{X_{x}}^{N} Y-\nabla_{X_{x}}^{M} Y \equiv-H\left(X_{x}, Y_{x}\right),
\end{aligned}
$$

where $H$ is the second fundumental form; and

$$
Y \uparrow M \perp T M \Longrightarrow \mathcal{S}_{x}\left(X_{x}\right) Y_{x}=-\Pi_{x} \nabla_{X_{x}}^{N} Y .
$$

Thus, by choosing $Y \in T N$ so that either $Y \uparrow M \in T M$ or $Y \uparrow M \perp T M$, we see that

$$
\Pi_{x}^{\perp} \circ \mathcal{S}_{x}\left(X_{x}\right)=\mathcal{S}_{x}\left(X_{x}\right) \circ \Pi_{x} \text { and } \Pi_{x} \circ \mathcal{S}_{x}\left(X_{x}\right)=\mathcal{S}_{x}\left(X_{x}\right) \circ \Pi_{x}^{\perp} .
$$

In addition, if $Y_{x} \in T_{x} M$, then we can choose $X, Y \in T N$ which agree with $X_{x}$ and $Y_{x}$ at $x$ and satisfy $X \uparrow M, Y \uparrow M \in T M$. Hence, by (1.3),

$$
\mathcal{S}_{x}\left(X_{x}\right) Y_{x}-\mathcal{S}\left(Y_{x}\right) X_{x}=\Pi_{x}^{\perp}[X, Y]_{x}=0,
$$

since $\nabla^{N}$ and $\nabla^{M}$ are torsion free and $[X, Y]_{x} \in T_{x} M$. In other words,

$$
X_{x}, Y_{x} \in T_{x} M \Longrightarrow \mathcal{S}_{x}\left(X_{x}\right) Y_{x}=\mathcal{S}\left(Y_{x}\right) X_{x}
$$

Lemma 1.7. Given $x \in M$, define $a_{x} \in \operatorname{Hom}\left(T_{x} M ; \operatorname{Hom}\left(T_{x} N ; T_{x} N\right)\right)$ so that

$$
a_{x}\left(X_{x}\right)=\mathcal{S}_{x}\left(X_{x}\right) \circ\left(\Pi_{x}-\Pi_{x}^{\perp}\right) .
$$

Then $a_{x}\left(X_{x}\right)$ is skew symmetric on $T_{x} N$ for each $X_{x} \in T_{x} M$. Next, for $p \in C^{1}([0, \infty) ; M)$, determine

$$
t \in[0, \infty) \longmapsto O_{p}(t) \in \operatorname{Hom}\left(T_{p(0)} N ; T_{p(t)} N\right)
$$


by

$$
\frac{d}{d t}\left(\mathcal{T}_{p \uparrow[0, t]}^{N}\right)^{-1} \circ O_{p}(t)=\left(\mathcal{T}_{p \uparrow[0, t]}^{N}\right)^{-1} \circ a_{p(t)}(\dot{p}(t)) \circ O_{p}(t) \quad \text { with } O_{p}(0)=I .
$$

Then, for each $t \in[0, \infty), O_{p}(t)$ is unitary from $T_{p(0)} N$ onto $T_{p(t)} N$,

$$
\Pi_{p(t)} \circ O_{p}(t)=O_{p}(t) \circ \Pi_{p(0)} \quad \text { and } \quad \Pi_{p(t)}^{\perp} \circ O_{p}(t)=O_{p}(t) \circ \Pi_{p(0)}^{\perp} .
$$

In fact,

$$
O_{p}(t) \uparrow T_{p(0)} M=\mathcal{T}_{p \uparrow[0, t]}^{M}
$$

Proof. Clearly the skew symmetry follows from (1.5).

Next set $x=p(0)$, let $Y_{x} \in T_{N}$ be given, and set $Y(t)=O_{p}(t) Y_{x}$. Then

$$
\frac{D^{N}}{d t} Y(t)=\mathcal{T}_{p \uparrow[0, t]}^{N} \frac{d}{d t}\left(\mathcal{T}_{p \uparrow[0, t]}^{N}\right)^{-1} Y(t)=a_{p(t)}(\dot{p}(t)) Y(t),
$$

where $\frac{D^{N}}{d t}$ denotes $N$-covariant differentiation along $p$. Hence, $t \in[0, \infty) \longmapsto$ $Y(t) \in T_{p(t)} N$ is characterized as the solution to

$$
\frac{D^{N}}{d t} Y(t)=a_{p(t)}(\dot{p}(t)) Y(t) \quad \text { with } Y(0)=Y_{x}
$$

In particular, because of the skew symmetry of $a_{p(t)}(\dot{p}(t))$,

$$
\frac{d}{d t}\|Y(t)\|^{2}=2\left\langle\frac{D^{N}}{d t} Y(t), Y(t)\right\rangle=0
$$

and so $O_{p}(t)$ is unitary. Now set $\tilde{Y}(t)=\Pi_{p(t)} Y(t)$. Then

$$
\frac{D^{N}}{d t} \tilde{Y}(t)=\mathcal{S}_{p(t)}(\dot{p}(t)) Y(t)+\Pi_{p(t)} a_{p(t)}(\dot{p}(t)) Y(t)=a_{p(t)}(\dot{p}(t)) \tilde{Y}(t),
$$

where, in the last step, we have again applied (1.5). Thus, by the characterization given in $\left(^{*}\right)$, we see that $\tilde{Y}(t)=O_{p}(t) \tilde{Y}(0)$. From this it follows immediately that $\Pi_{p(t)} \circ O_{p}(t)=O_{p}(t) \circ \Pi_{x}$, and, obviously, $\Pi_{p(t)}^{\perp} \circ O_{p}(t)=$ $O_{p}(t) \circ \Pi_{x}^{\perp}$ comes along for free. Finally, to prove that $Y(t)=\mathcal{T}_{p \uparrow[0, t]}^{M} Y_{x}$ when $Y_{x} \in T_{x} M$, simple observe that, because $Y(t) \in T_{p(t)} M$,

$$
\frac{D^{N}}{d t} Y(t)=a_{p(t)}(\dot{p}(t)) Y(t) \perp T_{p(t)} M
$$


follows from (1.5). In other words,

$$
\frac{D^{M}}{d t} Y(t)=\Pi_{p(t)} \frac{D^{N}}{d t} Y(t)=0,
$$

and this proves that $Y(t)=\mathcal{T}_{p \uparrow[0, t]}^{M} Y_{x}$.

Finally, we close this section with the observation that if $\varphi \in C^{2}(N ; \mathbb{R})$ and $x \in M$, then

$$
\begin{aligned}
& X_{x}, Y_{x} \in T_{x} M \Longrightarrow \\
& \quad\left\langle X_{x}, \operatorname{hess}_{x}^{M} \varphi Y_{x}\right\rangle=\left\langle X_{x}, \operatorname{hess}_{x}^{N} \varphi Y_{x}\right\rangle+\mathcal{S}_{x}\left(X_{x}\right) Y_{x} \varphi .
\end{aligned}
$$

To see this, simply recall that, for any extension $Y$ of $Y_{x}$ to $N$ with $Y \uparrow M \in$ $T M$,

$$
\begin{aligned}
\left\langle X_{x}, \operatorname{hess}_{x}^{M} \varphi Y_{x}\right\rangle & =X_{x} Y \varphi-\nabla_{X}^{M} Y \varphi=X_{x} Y \varphi-\nabla_{X}^{N} Y \varphi+\mathcal{S}_{x}\left(X_{x}\right) Y_{x} \varphi \\
& =\left\langle X_{x}, \operatorname{hess}_{x}^{N} \varphi Y_{x}\right\rangle+\mathcal{S}_{x}\left(X_{x}\right) Y_{x} \varphi .
\end{aligned}
$$

As a consequence of (1.9) and the representation of Laplacian as the trace of the Hessian, we obtain

$$
\Delta^{M} \varphi=\operatorname{Trace}^{M}\left(\operatorname{hess}_{x}^{N} \varphi\right) \varphi-B \varphi,
$$

where, for each $x \in M$ and orthonormal basis $\left(\left(E_{1}\right)_{x}, \ldots,\left(E_{n}\right)_{m}\right)$ in $T_{x} N$,

$$
B_{x} \equiv-\sum_{i=1}^{m} \mathcal{S}\left(\Pi_{x}\left(E_{i}\right)_{x}\right) \Pi_{x}\left(E_{i}\right)_{x}=\sum_{i=1}^{m} H\left(\Pi_{x}\left(E_{i}\right)_{x}, \Pi_{x}\left(E_{i}\right)_{x}\right)
$$

is (apart from normalization) the mean curvature vector (cf. page 49 in [2]).

\section{Moving to the Orthonormal Frame Bundle.}

In this section we will interpret the results of $\S 1$ in terms of the orthonormal frame bundle (cf. Chapter 8 of [3] for a treatment using the notation adopted here or [1] for a thorough treatment) $\mathcal{O}(N)$ over $N$. That is, elements $\mathfrak{f}$ of $\mathcal{O}(N)$ are frames $\left(x, \mathfrak{e}_{x}\right)$, where $x \in N$ and $\mathfrak{e}_{x}=\left(\left(E_{1}\right)_{x}, \ldots,\left(E_{n}\right)_{x}\right)$ is an orthonormal basis in $T_{x} N$. We use $\pi: \mathcal{O}(N) \longrightarrow N$ to denote the fiber map $\pi \mathfrak{f}=x$, and, for convenience, we identify $\mathfrak{f}$ with the isometry from $\mathbb{R}^{n}$ onto $T_{x} N$ given by

$$
\mathfrak{f} \boldsymbol{\xi}=\sum_{i=1}^{n} \xi_{i}\left(E_{i}\right)_{x} \quad \text { for } \boldsymbol{\xi}=\left(\xi_{1}, \ldots, \xi_{n}\right) \in \mathbb{R}^{n}
$$


Next, recall that $\mathcal{O}(N)$ is a principle bundle over $N$ with fiber the orthogonal group $\mathcal{O}\left(\mathbb{R}^{n}\right)$, and, for $O \in \mathcal{O}\left(\mathbb{R}^{n}\right)$, let $R_{O}: \mathcal{O}(N) \longrightarrow \mathcal{O}(N)$ be the map defined so that $\left(R_{O} \mathfrak{f}\right)=\mathfrak{f}(O \boldsymbol{\xi})$ for $\mathfrak{f} \in \mathcal{O}(N)$ and $\boldsymbol{\xi} \in \mathbb{R}^{n}$. Further, given $a \in o\left(\mathbb{R}^{n}\right)$, define the vertical vector field $\lambda(a)$ on $\mathcal{O}(N)$ so that

$$
\lambda(a)_{\mathfrak{f}}=\left.\frac{d}{d s} R_{e^{s a \mathfrak{f}}}\right|_{s=0} ;
$$

and, given $\boldsymbol{\xi} \in \mathbb{R}^{n}$, define the canonical horizontal vector field $\mathfrak{E}(\boldsymbol{\xi})$ on $\mathcal{O}(N)$ so that $\mathfrak{E}(\boldsymbol{\xi})_{\mathfrak{f}}$ is the horizontal lift to $\mathfrak{f}$ of $\mathfrak{f} \boldsymbol{\xi} \in T_{x} M$. Finally, the solder form $\phi$ and connection 1-from $\omega$ are defined (cf. page 181 in [3]) on $T_{\mathfrak{f}} \mathcal{O}(N)$ into $\mathbb{R}^{n}$ and $o\left(\mathbb{R}^{n}\right)$, respectively, so that

$$
\mathfrak{X}_{\mathfrak{f}}=\mathfrak{E}\left(\phi\left(\mathfrak{X}_{\mathfrak{f}}\right)\right)_{\mathfrak{f}}+\lambda\left(\omega\left(\mathfrak{X}_{\mathfrak{f}}\right)\right)_{\mathfrak{f}}
$$

gives the resolution of $\mathfrak{X}_{\mathfrak{f}}$ into its horizontal and vertical components.

Define $\hat{\Pi}: \pi^{-1}(M) \longrightarrow \operatorname{Hom}\left(\mathbb{R}^{n} ; \mathbb{R}^{n}\right)$ so that $\hat{\Pi}_{\mathfrak{f}}=\mathfrak{f}^{-1} \circ \Pi_{\pi(\mathfrak{f})} \circ \mathfrak{f}$. Clearly, for each $\mathfrak{f} \in \pi^{-1}(M), \hat{\Pi}_{\mathfrak{f}}$ is the orthogonal projection onto the subspace of $\boldsymbol{\xi} \in \mathbb{R}^{n}$ such that $\mathfrak{f} \boldsymbol{\xi} \in T_{\pi(\mathfrak{f})} M$. By using the fact (cf. (8.22) in [3]) that, for any vector field $Y$ on $N$,

$$
\mathfrak{f}^{-1}\left(\nabla_{\mathfrak{f} \xi}^{N} Y\right)=\mathfrak{E}(\boldsymbol{\xi})_{\mathfrak{f}} \Xi_{Y}, \quad \text { where } \Xi_{Y}(\mathfrak{f}) \equiv \mathfrak{f}^{-1} Y_{\pi \mathfrak{f}},
$$

we see that, for $\mathfrak{f} \xi \in T_{\pi(\mathfrak{f})} M$,

$$
\mathfrak{f}^{-1} \nabla_{\mathfrak{f} \boldsymbol{\xi}}^{N}(\Pi Y)=\left(\mathfrak{E}(\boldsymbol{\xi})_{\mathfrak{f}} \hat{\Pi}\right) \mathfrak{f}^{-1} Y_{\pi \mathfrak{f}}+\hat{\Pi}_{\mathfrak{f}}\left(\mathfrak{f}^{-1} \nabla_{\mathfrak{f} \mathfrak{\xi}}^{N} Y\right) .
$$

Hence, by (1.2),

$$
\begin{aligned}
& \hat{\mathcal{S}}_{\mathfrak{f}}(\boldsymbol{\xi}) \equiv \mathfrak{f}^{-1} \circ \mathcal{S}_{\pi \mathfrak{f}}(\mathfrak{f} \boldsymbol{\xi}) \circ \mathfrak{f}=\mathfrak{E}(\boldsymbol{\xi})_{\mathfrak{f}} \hat{\Pi} \\
& \qquad \text { for } \mathfrak{f} \in \pi^{-1}(M) \text { and } \boldsymbol{\xi} \in \mathfrak{f}^{-1}\left(T_{\pi \mathfrak{f}} M\right) .
\end{aligned}
$$

Next, observe that $\pi^{-1}(M)$ is a submanifold of $\mathcal{O}(N)$. In fact, for $\mathfrak{f} \in$ $\pi^{-1}(M)$ and $\mathfrak{X}_{\mathfrak{f}} \in T_{\mathfrak{f}} \mathcal{O}(N), \mathfrak{X}_{\mathfrak{f}} \in T_{\mathfrak{f}}\left(\pi^{-1}(M)\right)$ if and only if $\hat{\Pi}_{\mathfrak{f}}^{\perp} \phi\left(\mathfrak{X}_{\mathfrak{f}}\right)=0$. Thus, for each $\boldsymbol{\xi} \in \mathbb{R}^{n}$,

$$
\mathfrak{f} \in \pi^{-1}(M) \longmapsto \hat{\mathfrak{E}}(\boldsymbol{\xi})_{\mathfrak{f}} \equiv \mathfrak{E}\left(\hat{\Pi}_{\mathfrak{f}} \boldsymbol{\xi}\right)_{\mathfrak{f}} \in T_{\mathfrak{f}} \mathcal{O}(N)
$$

is a vector field on $\pi^{-1}(M)$. Furthermore, if $\varphi \in C^{2}(N ; \mathbb{R})$, then, by $(2.2)$,

$$
\hat{\mathfrak{E}}(\boldsymbol{\xi})_{\mathfrak{f}} \circ \hat{\mathfrak{E}}(\boldsymbol{\eta})(\varphi \circ \pi)=\mathfrak{E}\left(\Pi_{\mathfrak{f}} \boldsymbol{\xi}\right)_{\mathfrak{f}} \circ \mathfrak{E}\left(\Pi_{\mathfrak{f}} \boldsymbol{\eta}\right)(\varphi \circ \pi)+\mathfrak{E}\left(\hat{\mathcal{S}}_{\mathfrak{f}}(\boldsymbol{\xi}) \boldsymbol{\eta}\right)_{\mathfrak{f}}(\varphi \circ \pi) .
$$


At the same time, because an alternative way to describe $\operatorname{hess}_{x}^{N} \varphi$ is to say that

$$
\operatorname{hess}_{x}^{N} \varphi Y_{x}=\nabla_{Y_{x}} \operatorname{grad}^{N} \varphi
$$

(2.1) leads to

$$
\left\langle\mathfrak{f} \hat{\Pi}_{\mathfrak{f}} \boldsymbol{\xi}, \operatorname{hess}_{\pi \mathfrak{f}}^{N} \varphi \mathfrak{f} \hat{\Pi}_{\mathfrak{f}} \boldsymbol{\eta}\right\rangle=\mathfrak{E}\left(\Pi_{\mathfrak{f}} \boldsymbol{\xi}\right)_{\mathfrak{f}} \circ \mathfrak{E}\left(\Pi_{\mathfrak{f}} \boldsymbol{\eta}\right)(\varphi \circ \pi) .
$$

Hence, after combining this with the preceding, (1.9) says that

$$
\left\langle\hat{\mathfrak{f}} \hat{\Pi}_{\mathfrak{f}} \boldsymbol{\xi}, \operatorname{hess}_{\pi \mathfrak{f}}^{M} \varphi \mathfrak{f} \hat{\Pi}_{\mathfrak{f}} \boldsymbol{\eta}\right\rangle=\hat{\mathfrak{E}}(\boldsymbol{\xi})_{\mathfrak{f}} \circ \hat{\mathfrak{E}}(\boldsymbol{\eta})(\varphi \circ \pi), \quad \mathfrak{f} \in \pi^{-1}(M)
$$

In particular, this means that for any orthonormal basis $\left(\mathbf{e}_{1}, \ldots, \mathbf{e}_{n}\right)$ in $\mathbb{R}^{n}$,

$$
\left(\Delta^{M} \varphi\right) \circ \pi=\sum_{i=1}^{n} \hat{\mathfrak{E}}\left(\mathbf{e}_{i}\right)^{2} \varphi \circ \pi \quad \text { on } \pi^{-1}(M)
$$

\section{Brownian Motion, an Extrinsic Approach.}

The formula (2.4) provides the basis for a construction of Brownian motion on $M$ via "projection" of the Brownian motion on $N$.

To see what we have in mind, recall (cf. $\S 8.2$ in [3]), one way to construct the Brownian motion on $N$ starting at a point $x$ is to roll a Euclidean Brownian motion (i.e., a Wiener process) on $T_{x} N$ onto $N$. That is, if $\mathbf{w}$ is a "piecewise smooth" Wiener path in $\mathbb{R}^{n}$ and $\mathfrak{f} \in \pi^{-1}(x)$, then we determine $\mathfrak{p}^{N}(\cdot, \mathfrak{f}, \mathbf{w})$ by

$$
\dot{\mathfrak{p}}^{N}(t, \mathfrak{f}, \mathbf{w})=\mathfrak{E}(\dot{\mathbf{w}})_{\mathfrak{p}^{N}(t, \mathfrak{f}, \mathbf{w})} \quad \text { with } \mathfrak{p}^{N}(0, \mathfrak{f}, \mathbf{w})=\mathfrak{f}
$$

and set $p^{N}(t, \mathfrak{f}, \mathbf{w})=\pi \circ \mathfrak{p}^{N}(t, \mathfrak{f}, \mathbf{w})$. If almost every Wiener path were actually piecewise smooth, the distribution of $\mathbf{w} \rightsquigarrow p^{N}(\cdot, \mathfrak{f}, \mathbf{w})$ under Wiener measure would be the distribution of Brownian motion on $M$ starting at $x$. Because almost no Wiener is anywhere smooth, the preceding has to be interpretted by an appropriate limit procedure in which paths $\mathbf{w}$ are first replaced by polygononal approximations. The result of this procedure is equivalent to saying that we want to take $\mathbf{w} \rightsquigarrow \mathfrak{p}^{N}(\cdot, \mathfrak{f}, \mathbf{w})$ to be the solution to the Stratonovich stochastic differential equation

$$
d \mathfrak{p}^{N}(t, \mathfrak{f}, \mathbf{w})=\sum_{i=1}^{n} \mathfrak{E}\left(\mathbf{e}_{i}\right)_{\mathfrak{p}^{N}(t, \mathfrak{f}, \mathbf{w})} \circ d\left(\mathbf{e}_{i}, \mathbf{w}(t)\right) \quad \text { with } \mathfrak{p}^{N}(0, \mathfrak{f}, \mathbf{w})=\mathfrak{f}
$$


Indeed, if one ignores problems coming from possible explosion, Itô's formula for Stratonovich calculus says that, for any $\Phi \in C_{\mathrm{c}}^{2}(\mathcal{O}(N) ; \mathbb{R})$,

$$
\Phi\left(\mathfrak{p}^{N}(\cdot, \mathfrak{f}, \mathbf{w})\right)-\int_{0}^{t}\left(\frac{1}{2} \sum_{1}^{n} \mathfrak{E}\left(\mathbf{e}_{i}\right)_{\mathfrak{p}^{N}(\tau, \mathfrak{f}, \mathbf{w})}^{2} \Phi\right) d \tau
$$

is a martingale under Wiener measure. Thus, since, by another application of $(2.1)$,

$$
\left(\Delta^{N} \varphi\right) \circ \pi=\sum_{i=1}^{n} \mathfrak{E}\left(\mathbf{e}_{i}\right)^{2}(\varphi \circ \pi)
$$

it is clear that

$$
\varphi\left(p^{N}(t, \mathfrak{f}, \mathbf{w})\right)-\int_{0}^{t}\left(\frac{1}{2} \Delta^{N} \varphi\right)\left(p^{N}(\tau, \mathfrak{f}, \mathbf{w})\right) d \tau
$$

is a martingale for each $\varphi \in C_{\mathrm{c}}^{2}(N ; \mathbb{R})$. In other words, w $\rightsquigarrow p^{N}(\cdot, \mathfrak{f}, \mathbf{w})$ under Wiener measure has the distribution of a Brownian motion on $N$ starting at $x$. Moreover, because $\mathfrak{p}^{N}(\cdot, \mathfrak{f}, \mathbf{w})$ is the horizontal lift of $p^{N}(\cdot, \mathfrak{f}, \mathbf{w})$ to $\mathfrak{f}$ when $\mathbf{w}$ is piecewise smooth, it is reasonable to say that horizontal transport along the Brownian curve $p^{N}(\cdot, \mathfrak{f}, \mathbf{w}) \uparrow[0, t]$ is given by $\mathfrak{p}^{N}(t, \mathfrak{f}, \mathbf{w}) \circ \mathfrak{f}^{-1}$ even when $\mathbf{w}$ is a generic Wiener path.

With the preceding in mind, we now suppose that $x \in M$ and consider the Stratonovich stochastic differential equation

$$
d \mathfrak{q}^{M}(t, \mathfrak{f}, \mathbf{w})=\sum_{i=1}^{n} \hat{\mathfrak{E}}\left(\mathbf{e}_{i}\right)_{\mathfrak{q}^{M}(t, \mathfrak{f}, \mathbf{w})} \circ d\left(\mathbf{e}_{i}, \mathbf{w}(t)\right) .
$$

By precisely the same arguement as above, only this time using (2.4), we see that $\mathbf{w} \rightsquigarrow q^{M}(\cdot, \mathfrak{f}, \mathbf{w}) \equiv \pi \circ \mathfrak{q}^{M}(\cdot, \mathfrak{f}, \mathbf{w})$ is distributed under Wiener measure like a Brownian motion on $M$ starting at $x$. Furthermore, it is again reasonable to think of $\mathfrak{q}^{M}(\cdot, \mathfrak{f}, \mathbf{w})$ as the horizontal lift to $\mathfrak{f}$ of $q^{N}(\cdot, \mathfrak{f}, \mathbf{w})$. Thus, $\mathfrak{q}^{M}(t, \mathfrak{f}, \mathbf{w}) \circ \mathfrak{f}^{-1}$ gives parallel transport along $q^{M}(\cdot, \mathfrak{f}, \mathbf{w}) \uparrow[0, t]$ as a path in $N$. However, it does not give parallel transport along $q^{M}(\cdot, \mathbf{w})$ as a path in $M$. Indeed, it will seldom even take $T_{x} M$ into $T_{q^{M}(t, \mathfrak{f}, \mathbf{w})} M$.

A Remark about Explosion: In the preceding discussion, we ignored the question of explosion. Because we are assuming that $M$ is imbedded in $N$, we can (cf. Theorem 3.64 in [3]) show that explosion never occurs if we can check that, in the sense of distributions, $\Delta^{M} \rho \leq C(1+\rho)$ on $M$ for some $C<\infty$, where $\rho(y)=\operatorname{dist}^{N}(x, y)^{2}$ and $\operatorname{dist}^{N}(x, y)$ denotes the Riemannian 
distance in $N$ between $x$ and $y$. In view of (1.10), this is tantamount to testing whether

$$
\operatorname{Trace}^{M}\left(\operatorname{hess}^{N} \rho\right)-B \rho \leq C(1+\rho)
$$

in the sense of distributions. By the arguement in $\S 8.4$ of [3], the first term on the left can be handled if there exists an $\alpha>0$ such that

$$
\begin{aligned}
& \sum_{i=1}^{m}\left\langle R^{N}\left(Y_{y},\left(E_{i}\right)_{y}\right)\left(E_{i}\right)_{y}, Y_{y}\right\rangle \geq-\alpha(1+\rho(y))\left\|Y_{y}\right\|^{2} \\
& \text { for } y \in M \text { and } Y_{y} \in T_{y} N,
\end{aligned}
$$

where $\left(\left(E_{1}\right)_{y}, \ldots,\left(E_{m}\right)_{y}\right)$ is used to denote an orthonormal basis in $T_{y} M$. Thus, if such an $\alpha$ exists, then non-explosion is guaranteed by the existence of a $\beta>0$ such that $\left\langle B, \operatorname{grad}^{N} \rho\right\rangle \geq-\beta(1+\rho)$.

\section{A Second, and More Geometrically Sound, Appraoch.}

As we pointed out, although the $q^{M}(\cdot, \mathfrak{f}, \mathbf{w})$ is indeed Brownian motion on $M$ starting at $\pi \mathfrak{f}, \mathfrak{q}^{M}(\cdot, \mathfrak{f}, \mathbf{w})$ is the wrong lift of $q^{M}(\cdot, \mathfrak{f}, \mathbf{w})$ to $\mathfrak{f}$ if one is interested in parallel transport in $M$, as opposed to $N$. In addition, because our construction of the $m$-dimensional Brownian path $q^{M}(\cdot, \mathfrak{f}, \mathbf{w})$ used the $n$-dimensional Wiener path $\mathbf{w}$, one suspects that there should be a tighter construction of Brownian motion on $M$ : a construction which involves only an $m$-dimensional Wiener path.

Motivated by the preceding comments, we will now take a different tack. To understand the origins of this new approach, set (cf. (2.2))

$$
\hat{a}_{\mathfrak{f}}(\boldsymbol{\xi}) \equiv \hat{\mathcal{S}}_{\mathfrak{f}}\left(\hat{\Pi}_{\mathfrak{f}} \boldsymbol{\xi}\right) \circ\left(\hat{\Pi}_{\mathfrak{f}}-\hat{\Pi}_{\mathfrak{f}}^{\perp}\right)=\mathfrak{f}^{-1} \circ a_{\pi \mathfrak{f}}\left(\Pi_{\pi \mathfrak{f}} \mathfrak{f} \boldsymbol{\xi}\right) \circ \mathfrak{f} \quad \text { for } \mathfrak{f} \in \pi^{-1}(M) \text {. }
$$

Using (1.9), it is a straight-forward matter to check that

$$
\begin{aligned}
& \left(\mathfrak{E}(\boldsymbol{\xi})+\lambda\left(\hat{a}_{\mathfrak{f}}(\boldsymbol{\xi})\right)\right)_{\mathfrak{f}} \circ(\mathfrak{E}(\boldsymbol{\eta})+\lambda(\hat{a}(\boldsymbol{\eta})))(\varphi \circ \pi) \\
& =\left\langle\mathfrak{f} \boldsymbol{\xi}, \operatorname{hess}_{\pi \mathfrak{f}}^{M} \varphi \mathfrak{f} \boldsymbol{\eta}\right\rangle \quad \text { for } \mathfrak{f} \in \pi^{-1}(M) \text { and } \boldsymbol{\xi}, \boldsymbol{\eta} \in \mathfrak{f}^{-1}\left(T_{\pi \mathfrak{f}} M\right) .
\end{aligned}
$$

Indeed, all that one needs to do is remember that vertical vectors kill $\varphi \circ \pi$, observe that

$$
\lambda\left(\hat{a}_{\mathfrak{f}}(\boldsymbol{\xi})\right)_{\mathfrak{f}} \circ \mathfrak{E}(\boldsymbol{\eta})=\mathfrak{E}\left(\hat{a}_{\mathfrak{f}}(\boldsymbol{\xi}) \boldsymbol{\eta}\right)_{\mathfrak{f}}+\mathfrak{E}(\boldsymbol{\xi})_{\mathfrak{f}} \circ \lambda\left(\hat{a}_{\mathfrak{f}}(\boldsymbol{\eta})\right),
$$

and note that, because $\hat{\Pi}_{\mathfrak{f}}^{\perp} \boldsymbol{\xi}=0=\hat{\Pi}_{\mathfrak{f}}^{\perp} \boldsymbol{\eta}, \hat{a}_{\mathfrak{f}}(\boldsymbol{\xi}) \boldsymbol{\eta}=\hat{\mathcal{S}}(\boldsymbol{\xi}) \boldsymbol{\eta}$. In particular, if $\left(\mathbf{e}_{1}, \ldots, \mathbf{e}_{m}\right)$ is an orthonormal basis for $\mathfrak{f}^{-1}\left(T_{\pi \mathfrak{f}} M\right)$, then

$$
\left(\Delta_{M} \varphi\right) \circ \pi(\mathfrak{f})=\sum_{i=1}^{m}\left(\mathfrak{E}\left(\mathbf{e}_{i}\right)+\lambda\left(\hat{a}\left(\mathbf{e}_{i}\right)\right)_{\mathfrak{f}}^{2}(\varphi \circ \pi) .\right.
$$


In order to base a construction of Brownian motion on (4.3), we will make use of the information contained in the following simple lemmas.

Lemma 4.4. Given a piecewise continuously differentiable, continuous $\mathfrak{q}$ : $[0, \infty) \longrightarrow \pi^{-1}(M)$, determine $t \in[0, \infty) \longmapsto \hat{O}_{\mathfrak{q}}(t) \in \operatorname{Hom}\left(\mathbb{R}^{n} ; \mathbb{R}^{n}\right)$ by

$$
\frac{d}{d t} \hat{O}_{\mathfrak{q}}(t)=\hat{a}_{\mathfrak{q}(t)}\left(\phi(\dot{\mathfrak{q}}(t)) \hat{O}_{\mathfrak{q}}(t) \quad \text { with } \hat{O}_{\mathfrak{q}}(0)=I\right. \text {. }
$$

Then $\hat{O}_{\mathfrak{q}}(t)$ is an element of the orthognal group $\mathcal{O}\left(\mathbb{R}^{n}\right)$ for each $t \in[0, \infty)$. Moreover, if $\mathfrak{q}$ is horizontal (i.e., $\omega(\dot{\mathfrak{q}}(t)) \equiv 0$ ), then (cf. Lemma 1.7)

$$
\mathfrak{q}(t) \circ \hat{O}_{\mathfrak{q}}(t) \circ \mathfrak{q}(0)^{-1}=O_{\pi \circ \mathfrak{q}}(t), \quad t \in[0, \infty),
$$

and so

$$
\hat{\Pi}_{\mathfrak{q}(t)} \circ \hat{O}_{\mathfrak{q}}(t)=\hat{O}_{\mathfrak{q}}(t) \circ \hat{\Pi}_{\mathfrak{q}(0)}, \quad t \in[0, \infty) .
$$

Proof. Without loss in generality, we will assume that $\mathfrak{q}$ is continuous differentiable everywhere.

Because (cf. the first part of Lemma 1.7) the values of $\hat{a}$ are always in the Lie algebra $o\left(\mathbb{R}^{n}\right)$ of skew symmetric operators, it is trivial to check that $\hat{O}_{\mathfrak{q}}(t) \in \mathcal{O}\left(\mathbb{R}^{n}\right)$ for all $t \geq 0$. To check (4.6) when $\mathfrak{q}$ is horizontal, let $\boldsymbol{\xi} \in \mathbb{R}^{n}$ be given, and set $X(t)=\mathfrak{q}(t) \hat{O}_{\mathfrak{q}}(t) \boldsymbol{\xi} \in T_{\pi \mathfrak{q}(t)} M$. Then, because $\mathfrak{q}$ is horizontal,

$$
\frac{D^{N}}{d t} X(t)=\mathfrak{q}(t) \frac{d}{d t} \hat{O}_{\mathfrak{q}}(t) \boldsymbol{\xi}=\mathfrak{q}(t) \hat{a}_{\mathfrak{q}(t)}\left(\phi(\dot{\mathfrak{q}}(t)) \hat{O}_{\mathfrak{q}}(t) \boldsymbol{\xi}=a\left((\pi \circ \mathfrak{q})^{\cdot}(t)\right) X(t) .\right.
$$

Since this means that $X(t)=O_{\pi \circ \mathfrak{q}}(t) \mathfrak{q}(0) \boldsymbol{\xi}$, (4.6) follows. Finally, (4.7) is immediate from (4.6) and the corresponding fact (cf. the last part of Lemma 1.7) for $O_{\pi \circ \mathfrak{q}}(t)$.

Lemma 4.8. Let $\mathfrak{p} \in C\left([0, \infty) ; \pi^{-1}(M)\right)$ be a piecewise continuously differentiable, set $p=\pi \circ \mathfrak{p}$, and let $\mathfrak{q}$ be the horizontal lift of $p$ to $\mathfrak{p}(0)$. Then the following are equivalent:

(1) $\omega(\dot{\mathfrak{p}}(t))=\hat{a}_{\mathfrak{p}(t)}(\phi(\dot{\mathfrak{p}}(t)))$ for all $t \geq 0$ at which $\mathfrak{p}$ is continuously differentiable,

(2) $\mathfrak{p}(t)=R_{\hat{O}_{\mathfrak{q}}(t)} \mathfrak{q}(t)$,

(3) $O_{p}(t)=\mathfrak{p}(t) \mathfrak{p}(0)^{-1}$. 
In particular, any one of these implies that $\hat{\Pi}_{\mathfrak{p}(t)}=\hat{\Pi}_{\mathfrak{p}(0)}$ for all $t \in[0, \infty)$.

Proof. Again we may and will assume that $\mathfrak{p}$ is continuously differentiable everywhere.

The equivalence of (2) and (3) just a restatement of (4.6), and the final conclusion is simply a restatement of (3). To prove the equivalence of (1) and (2), first note that

$$
(1) \Longleftrightarrow \dot{\mathfrak{p}}(t)=\mathfrak{E}\left(\mathfrak{p}(t)^{-1} \dot{p}(t)\right)_{\mathfrak{p}(t)}+\lambda\left(\hat{a}_{\mathfrak{p}(t)}\left(\mathfrak{p}(t)^{-1} \dot{p}(t)\right)\right)_{\mathfrak{p}(t)} .
$$

Thus, it suffices to show that if $\mathfrak{r}(t) \equiv R_{\hat{O}_{\mathfrak{q}}(t)} \mathfrak{q}(t)$, then

$$
\dot{\mathfrak{r}}(t)=\mathfrak{E}\left(\mathfrak{r}(t)^{-1} \dot{p}(t)\right)_{\mathfrak{r}(t)}+\lambda\left(\hat{a}_{\mathfrak{r}(t)}\left(\mathfrak{r}(t)^{-1} \dot{p}(t)\right)\right)_{\mathfrak{r}(t)}
$$

But $\dot{\mathfrak{r}}(t)$ is equal to

$$
\begin{array}{rl}
\left(R_{\hat{O}_{\mathfrak{q}}(t)}\right)_{*} & \mathfrak{E}\left(\mathfrak{q}(t)^{-1} \dot{p}(t)\right)_{\mathfrak{q}(t)}+\lambda\left(\hat{O}_{\mathfrak{q}}(t)^{\top} \hat{a}_{\mathfrak{q}(t)}\left(\mathfrak{q}(t)^{-1} \dot{p}(t)\right) \hat{O}_{\mathfrak{q}}(t)\right)_{\mathfrak{r}(t)} \\
& =\mathfrak{E}\left(\mathfrak{r}(t)^{-1} \dot{p}(t)\right)_{\mathfrak{r}(t)}+\lambda\left(\hat{a}_{\mathfrak{r}(t)}\left(\mathfrak{r}(t)^{-1} \dot{p}(t)\right)\right)_{\mathfrak{r}(t)} .
\end{array}
$$

In order to bring out the goemetric content of the preceding lemmas, we think of $\mathbb{R}^{m}$ as the subspace of $\boldsymbol{\xi}=\left(\xi_{1}, \ldots, \xi_{n}\right) \in \mathbb{R}^{n}$ such that $\xi_{i}=0$ for $m<i \leq n$, take $\hat{\Pi}^{0}$ to be orthogonal projection from $\mathbb{R}^{n}$ onto $\mathbb{R}^{m}$, and introduce the space

$$
\mathcal{O}^{N}(M) \equiv\left\{\mathfrak{f} \in \pi^{-1}(M): \hat{\Pi}_{\mathfrak{f}}=\hat{\Pi}^{0}\right\} .
$$

It should be clear that $\mathcal{O}^{N}(M)$ is submanifold of $\mathcal{O}(N)$. In fact, it is subbundle whose base is $M$ and fiber is

$$
\mathcal{O}^{\mathbb{R}^{n}}\left(\mathbb{R}^{m}\right) \equiv\left\{O \in \mathcal{O}\left(\mathbb{R}^{n}\right): \hat{\Pi}^{0} \circ O=O \circ \hat{\Pi}^{0}\right\}
$$

To see this, let $x \in M$ be given and note that there exists an open neighborhood $U$ of $x$ in $N$ on which there exist vector fields $E_{i}, 1 \leq i \leq n$, such that $\left(\left(E_{1}\right)_{y}, \ldots,\left(E_{n}\right)_{y}\right)$ is an orthonormal basis in $T_{y}(N)$ for all $y \in U$ and $\left(E_{i}\right)_{y} \in T_{y} M$ for all $y \in U \cap M$ and $1 \leq i \leq m$. Now set

$$
\mathfrak{f}_{y}=\left(y,\left(\left(E_{1}\right)_{y}, \ldots,\left(E_{n}\right)_{y}\right)\right),
$$


and observe that

$$
\begin{aligned}
(y, O) \in U \times \mathcal{O}\left(\mathbb{R}^{n}\right) & \longmapsto\left(y, R_{O} \mathfrak{f}_{y}\right) \in \pi^{-1}(U) \\
(y, O) \in(U \cap M) \times \mathcal{O}^{\mathbb{R}^{n}}\left(\mathbb{R}^{m}\right) & \longmapsto\left(y, R_{O} \mathfrak{f}_{y}\right) \in \pi^{-1}(U) \cap \mathcal{O}^{N}(M)
\end{aligned}
$$

are homeomorphic. Finally, let $o^{\mathbb{R}^{n}}\left(\mathbb{R}^{m}\right)$ denote the Lie algebra of $a \in o\left(\mathbb{R}^{n}\right)$ such that $\hat{\Pi}^{0} a=a \hat{\Pi}^{0}$, and note that $o^{\mathbb{R}^{n}}\left(\mathbb{R}^{m}\right)$ is the Lie algebra for the Lie $\operatorname{group} \mathcal{O}^{\mathbb{R}^{n}}\left(\mathbb{R}^{m}\right)$.

Lemma 4.9. If $\mathfrak{f} \in \mathcal{O}^{N}(M)$ and $a \in o\left(\mathbb{R}^{n}\right)$, then $\lambda(a)_{\mathfrak{f}} \in T_{\mathfrak{f}} \mathcal{O}^{N}(M)$ if and only if $a \in o^{\mathbb{R}^{n}}\left(\mathbb{R}^{m}\right)$. Moreover, if

$$
\mathfrak{E}^{M}(\boldsymbol{\xi})_{\mathfrak{f}}=\mathfrak{E}(\boldsymbol{\xi})_{\mathfrak{f}}+\lambda\left(\hat{a}_{\mathfrak{f}}(\boldsymbol{\xi})\right)_{\mathfrak{f}} \quad \text { for } \mathfrak{f} \in \mathcal{O}^{N}(M) \text { and } \boldsymbol{\xi} \in \mathbb{R}^{m},
$$

then $\mathfrak{E}^{M}(\boldsymbol{\xi})_{\mathfrak{f}} \in T_{\mathfrak{f}} \mathcal{O}^{N}(M)$. Finally, for $\mathfrak{f} \in \mathcal{O}^{N}(M)$ and $\mathfrak{X}_{\mathfrak{f}} \in T_{\mathfrak{f}} \mathcal{O}(N)$, $\mathfrak{X}_{\mathfrak{f}} \in T_{\mathfrak{f}} \mathcal{O}^{N}(M)$ if and only if

$$
\phi\left(\mathfrak{X}_{\mathfrak{f}}\right) \in \mathbb{R}^{m} \quad \text { and } \quad \omega^{M}\left(\mathfrak{X}_{f}\right) \equiv \omega\left(\mathfrak{X}_{\mathfrak{f}}\right)-\hat{a}_{\mathfrak{f}}\left(\phi\left(\mathfrak{X}_{\mathfrak{f}}\right)\right) \in o^{\mathbb{R}^{n}}\left(\mathbb{R}^{m}\right),
$$

in which case $\boldsymbol{\xi}=\phi\left(\mathfrak{X}_{\mathfrak{f}}\right)$ and $a=\omega^{M}\left(\mathfrak{X}_{\mathfrak{f}}\right)$ are the unique elements of $\mathbb{R}^{m}$ and $o^{\mathbb{R}^{n}}\left(\mathbb{R}^{m}\right)$, respectively, such that $\mathfrak{X}_{\mathfrak{f}}=\mathfrak{E}^{M}(\boldsymbol{\xi})_{\mathfrak{f}}+\lambda(a)_{\mathfrak{f}}$.

Proof. Let $\mathfrak{f} \in \mathcal{O}^{N}(M)$. The fact that $a \in o\left(\mathbb{R}^{n}\right)$ is an element of $o^{\mathbb{R}^{n}}\left(\mathbb{R}^{m}\right)$ if and only if $\lambda(a)_{\mathfrak{f}} \in T_{\mathfrak{f}} \mathcal{O}^{N}(M)$ is just a restatement of the fact that $o^{\mathbb{R}^{n}}\left(\mathbb{R}^{m}\right)$ is the Lie algebra for $\mathcal{O}^{\mathbb{R}^{n}}\left(\mathbb{R}^{m}\right)$. Next let $\boldsymbol{\xi} \in \mathbb{R}^{m}$. To see that $\mathfrak{E}^{M}(\boldsymbol{\xi})_{\mathfrak{f}} \in$ $T_{\mathfrak{f}} \mathcal{O}^{N}(M)$, we need only find a continuously differentiable $\mathfrak{p}:[0, \infty) \longrightarrow$ $\mathcal{O}^{N}(M)$ such that $\mathfrak{p}(0)=\mathfrak{f}$ and $\dot{\mathfrak{p}}(0)=\mathfrak{E}^{M}(\boldsymbol{\xi})_{\mathfrak{f}}$. To this end, determine $\mathfrak{p}:[0, \infty) \longrightarrow \pi^{-1}(M)$ by

$$
\dot{\mathfrak{p}}(t)=\hat{\mathfrak{E}}(\boldsymbol{\xi})_{\mathfrak{p}(t)}+\lambda\left(\hat{a}_{\mathfrak{p}(t)}(\boldsymbol{\xi})\right)_{\mathfrak{p}(t)} \quad \text { with } \mathfrak{p}(0)=\mathfrak{f} .
$$

Clearly $\dot{\mathfrak{p}}(0)=\mathfrak{E}^{M}(\boldsymbol{\xi})_{\mathfrak{f}}$. In addition, $\omega(\dot{\mathfrak{p}}(t))=\hat{a}_{\mathfrak{p}(t)}(\phi(\dot{\mathfrak{p}}(t))$ for all $t \geq 0$, and so, by the last part of Lemma $4.8, \hat{\Pi}_{\mathfrak{p}(t)}=\hat{\Pi}_{\mathfrak{p}(0)}=\hat{\Pi}^{0}$ for all $t \geq 0$. Thus $\mathfrak{p}(t) \in \mathcal{O}^{N}(M)$ for all $t \geq 0$.

To complete the proof from here, let $\mathfrak{X}_{\mathfrak{f}} \in T_{\mathfrak{f}} \mathcal{O}(N)$ be given. Then $\boldsymbol{\xi}=\phi\left(\mathfrak{X}_{\mathfrak{f}}\right)$ and $b=\omega\left(\mathfrak{X}_{\mathfrak{f}}\right)$ are the unique elements of $\mathbb{R}^{n}$ and $o\left(\mathbb{R}^{n}\right)$ such that $\mathfrak{X}_{\mathfrak{f}}=\mathfrak{E}(\boldsymbol{\xi})_{\mathfrak{f}}+\lambda(a)_{\mathfrak{f}}$. Obviously, $\mathfrak{X}_{\mathfrak{f}} \in T_{\mathfrak{f}} \pi^{-1}(M)$ if and only if $\boldsymbol{\xi} \in \mathbb{R}^{m}$. Hence, if $\mathfrak{X}_{\mathfrak{f}} \in T_{\mathfrak{f}} \mathcal{O}^{N}(M)$, and we write

$$
\mathfrak{X}_{\mathfrak{f}}=\mathfrak{E}^{M}(\boldsymbol{\xi})_{\mathfrak{f}}+\lambda(b)_{\mathfrak{f}},
$$

then $a=b-\hat{a}_{\mathfrak{f}}(\boldsymbol{\xi})=\omega^{M}\left(\mathfrak{X}_{\mathfrak{f}}\right)$, and, because $\lambda(a)_{\mathfrak{f}}=\mathfrak{X}_{\mathfrak{f}}-\mathfrak{E}^{M}(\boldsymbol{\xi})_{\mathfrak{f}} \in T_{\mathfrak{f}} \mathcal{O}^{N}(M)$, $a \in o^{\mathbb{R}^{n}}\left(\mathbb{R}^{m}\right)$. Conversely, if $\mathfrak{X}_{\mathfrak{f}}=\mathfrak{E}^{M}(\boldsymbol{\xi})_{\mathfrak{f}}+\lambda(a)_{\mathfrak{f}}$ for some $\boldsymbol{\xi} \in \mathbb{R}^{m}$ and $a \in o^{\mathbb{R}^{n}}\left(\mathbb{R}^{m}\right)$, then $\mathfrak{X}_{\mathfrak{f}} \in T_{\mathfrak{f}} \mathcal{O}^{N}(M), \boldsymbol{\xi}=\phi\left(\mathfrak{X}_{\mathfrak{f}}\right)$, and $a=\omega^{M}\left(\mathfrak{X}_{\mathfrak{f}}\right)$. 
We are now in a position to achieve both the goals set at the opening of the section. Namely, let $\mathbf{w}(\cdot)$ be an $\mathbb{R}^{n}$-valued Wiener process, and choose $\left(\mathbf{e}_{1}, \ldots, \mathbf{e}_{n}\right)$ to be an orthonormal basis in $\mathbb{R}^{n}$ with $\mathbf{e}_{i} \in \mathbb{R}^{m}$ for $1 \leq i \leq m$. Given $\mathfrak{f} \in \mathcal{O}^{N}(M)$, determine $\mathfrak{p}^{M}(\cdot, \mathfrak{f}, \mathbf{w})$ by the Stratonovich stochastic differential equation

$$
d \mathfrak{p}^{M}(t, \mathfrak{f}, \mathbf{w})=\sum_{i=1}^{m} \mathfrak{E}^{M}\left(\mathbf{e}_{i}\right)_{\mathfrak{p}^{M}(t, \mathfrak{f}, \mathbf{w})} \circ d\left(\mathbf{e}_{i}, \mathbf{w}(t)\right) \quad \text { with } \mathfrak{p}^{M}(0, \mathfrak{f}, \mathbf{w})=\mathfrak{f} .
$$

(Notice that, although $\mathbf{w}$ is $\mathbb{R}^{n}$-valued, only the $\mathbb{R}^{m}$-component $\hat{\Pi}^{0} \mathbf{w}$ enters (4.10).) By the first part of Lemma 4.9, so long as it has not exploded, $t \rightsquigarrow \mathfrak{p}^{M}(t, \mathfrak{f}, \mathbf{w})$ takes its values in $\mathcal{O}^{N}(M)$. Furthermore, if $p^{M}(\cdot, \mathfrak{f}, \mathbf{w}) \equiv$ $\pi \circ \mathfrak{p}^{M}(\cdot, \mathfrak{f}, \mathbf{w})$, then, by $(4.3), \mathbf{w} \rightsquigarrow p^{M}(\cdot, \mathfrak{f}, \mathbf{w})$ is a Brownian motion on $M$ starting at $x \equiv \pi \mathfrak{f}$; and the fact that $\mathfrak{p}^{M}(t, \mathfrak{f}, \mathbf{w}) \in \mathcal{O}^{N}(M)$ becomes the statement that

$$
\Pi_{p^{M}(t, \mathfrak{f}, \mathbf{w})} \circ \mathfrak{p}^{M}(t, \mathfrak{f}, \mathbf{w})=\mathfrak{p}^{M}(t, \mathfrak{f}, \mathbf{w}) \circ \hat{\Pi}_{\mathfrak{f}} .
$$

In fact, by (3) in Lemma 4.8, we know that

$$
\mathfrak{p}^{M}(t, \mathfrak{f}, \mathbf{w}) \mathfrak{f}^{-1}=O_{p^{M}(\cdot, \mathfrak{f}, \mathbf{w})}(t)
$$

for piecewise smooth w's, and so, even when w is a generic Wiener path, we can use (4.11) to define $O_{p^{M}(\cdot, \mathfrak{f}, \mathbf{w})}(t)$, in which case it is clear that $O_{p^{M}(\cdot, \mathfrak{f}, \mathbf{w})}(t) \uparrow T_{\pi \mathfrak{f}} M$ gives us a notion of parallel transport along $p^{M}(\cdot, \mathfrak{f}, \mathbf{w}) \uparrow[0, t]$ as a path in $M$.

\section{The Relationship between the Constructions in $\S 3$ and $\S 4$.}

It may be useful to point out how $\mathfrak{p}^{M}(\cdot, \mathfrak{f}, \mathbf{w})$ and $\mathfrak{q}^{M}(\cdot, \mathfrak{f}, \mathbf{w})$ are related. The idea is that because (cf. Lemma 4.8) $t \rightsquigarrow R_{\hat{O}_{\mathfrak{q}}(t)}$ converts the horizontal lift $\mathfrak{q}$ to $\mathcal{O}(N)$ of a path $p:[0, \infty) \longrightarrow M$ into its horizontal lift to $\mathcal{O}^{N}(M)$, the same ought to be true for Brownian paths.

Thus, choose an orthonormal basis $\left(\mathbf{e}_{1}, \ldots, \mathbf{e}_{n}\right)$ in $\mathbb{R}^{n}$ so that $\mathbf{e}_{i} \in \mathbb{R}^{m}$ when $1 \leq i \leq m$. Next, given $\mathfrak{f} \in \mathcal{O}^{N}(M)$, determine $\mathfrak{q}^{M}(\cdot, \mathfrak{f}, \mathbf{w})$ relative to $\left(\mathbf{e}_{1}, \ldots, \mathbf{e}_{n}\right)$ by (3.1). At the same time, consider the Stratonovich stochastic differential equation

$$
\begin{aligned}
d \hat{O}(t, \mathfrak{f}, \mathbf{w})= & \sum_{i=1}^{n} \hat{a}_{\mathfrak{q}^{M}(t, \mathfrak{f}, \mathbf{w})}\left(\mathbf{e}_{i}\right) \hat{O}(t, \mathfrak{f}, \mathbf{w}) \circ d\left(\mathbf{e}_{i}, \mathbf{w}(t)\right) \\
& \text { with } \hat{O}(0, \mathfrak{f}, \mathbf{w})=I .
\end{aligned}
$$


Obviously, $\hat{O}(t, \mathfrak{f}, \mathbf{w})$ plays the role of $\hat{O}_{\mathfrak{q}^{M}(t, \mathfrak{f}, \mathbf{w})}$ for generic Wiener paths $\mathbf{w}$. Thus, by Lemma 4.8, we should expect that

$$
\mathbf{w} \rightsquigarrow \mathfrak{r}(\cdot, \mathfrak{f}, \mathbf{w}) \equiv R_{\hat{O}(\cdot, \mathfrak{f}, \mathbf{w})} \mathfrak{q}^{M}(\cdot, \mathfrak{f}, \mathbf{w})
$$

should have the same distribution as $\mathbf{w} \rightsquigarrow \mathfrak{p}^{M}(\cdot, \mathfrak{f}, \mathbf{w})$. (Indeed, at first, one might have guessed that $\mathfrak{r}(\cdot, \mathfrak{f}, \mathbf{w})$ would be even equal to $\mathfrak{p}^{M}(\cdot, \mathfrak{f}, \mathbf{w})$. However, as we are about to see, that guess ignores a required rotation of w.) To test this expectation, first note that, as a consequence of $(2) \Longleftrightarrow(3)$ in Lemma 4.8 , we know that $\mathfrak{r}(\cdot, \mathfrak{f}, \mathbf{w})$ is a path in $\mathcal{O}^{N}(M)$. Second, by Itô's formula for Stratonovich calculus,

$$
\begin{aligned}
d \mathfrak{r}(t, \mathfrak{f}, \mathbf{w}) & =\sum_{i=1}^{n}\left(\hat{\mathfrak{E}}\left(\hat{O}(t, \mathfrak{f}, \mathbf{w})^{\top} \mathbf{e}_{i}\right)_{\mathfrak{r}(t, \mathfrak{f}, \mathbf{w})}\right. \\
& \left.+\lambda\left(\hat{O}(t, \mathfrak{f}, \mathbf{w})^{\top} \hat{a}_{\mathfrak{q}^{M}(t, \mathfrak{f}, \mathbf{w})}\left(\mathbf{e}_{i}\right) \hat{O}(t, \mathfrak{f}, \mathbf{w})\right)\right)_{\mathfrak{r}(t, \mathfrak{f}, \mathbf{w})} \circ d\left(\mathbf{e}_{i}, \mathbf{w}(t)\right)
\end{aligned}
$$

Next observe that

$$
\hat{O}(t, \mathfrak{f}, \mathbf{w})^{\top} \hat{a}_{\mathfrak{q}^{M}(t, \mathfrak{f}, \mathbf{w})}\left(\mathbf{e}_{i}\right) \hat{O}(t, \mathfrak{f}, \mathbf{w})=\hat{a}_{\mathfrak{r}(t, \mathfrak{f}, \mathbf{w})}\left(\hat{O}(t, \mathfrak{f}, \mathbf{w})^{\top} \mathbf{e}_{i}\right) .
$$

Further, observe that $\hat{\Pi}_{\mathfrak{r}(t, \mathfrak{f}, \mathbf{w})}=\hat{\Pi}^{0}$ is equivalent to

$$
\hat{\Pi}_{\mathfrak{q}^{M}(t, \mathfrak{f}, \mathbf{w})} \hat{O}(t, \mathfrak{f}, \mathbf{w})=\hat{O}(t, \mathfrak{f}, \mathbf{w}) \hat{\Pi}^{0}
$$

Hence, we have now shown that

$$
\begin{aligned}
d \mathfrak{r}(t, \mathfrak{f}, \mathbf{w}) & =\sum_{i=1}^{m} \mathfrak{E}^{M}\left(\hat{O}(t, \mathfrak{f}, \mathbf{w})^{\top} \mathbf{e}_{i}\right)_{\mathfrak{r}(t, \mathfrak{f}, \mathbf{w})} \circ d\left(\mathbf{e}_{i}, \mathbf{w}(t)\right) \\
& =\sum_{i, j=1}^{m} \mathfrak{E}^{M}\left(\mathbf{e}_{j}\right)_{\mathfrak{r}(t, \mathfrak{f}, \mathbf{w})} \circ d\left(\mathbf{e}_{j}, \mathbf{W}(t)\right),
\end{aligned}
$$

where

$$
\mathbf{W}(t) \equiv \sum_{i=1}^{n} \int_{0}^{t}\left(\hat{O}(\tau, \mathfrak{f}, \mathbf{w})^{\top} \mathbf{e}_{i}, \mathbf{e}_{j}\right) \circ d\left(\mathbf{e}_{i}, \mathbf{w}(\tau)\right) .
$$

Hence, if we can check that $\hat{\Pi}^{0} \mathbf{W}=\hat{\Pi}^{0} \tilde{\mathbf{w}}$ where $\tilde{\mathbf{w}}$ is an $\mathbb{R}^{n}$-valued Wiener process, we will know that

$$
R_{O(\cdot, \mathfrak{f}, \mathbf{w})} \mathfrak{q}^{M}(\cdot, \mathfrak{f}, \mathbf{w})=\mathfrak{p}^{M}(\cdot, \mathfrak{f}, \tilde{\mathbf{w}})
$$


and therefore that $\mathbf{w} \rightsquigarrow R_{O(\cdot, \mathfrak{f}, \mathbf{w})} \mathfrak{q}^{M}(\cdot, \mathfrak{f}, \mathbf{w})$ does indeed have the same distribution as $\mathbf{w} \rightsquigarrow \mathfrak{p}^{M}(\cdot, \mathfrak{f}, \mathbf{w})$.

To see that $\hat{\Pi}^{0} \mathbf{W}$ is an $\mathbb{R}^{m}$-valued Wiener path, note that

$$
\begin{aligned}
d\left(\hat{\Pi}^{0} \mathbf{W}\right)= & \hat{\Pi}^{0} \hat{O}(t, \mathfrak{f}, \mathbf{w})^{\top} d \mathbf{w}(t) \\
& -\frac{1}{2}\left(\sum_{i=1}^{n} \hat{\Pi}^{0} \hat{O}(t, \mathfrak{f}, \mathbf{w})^{\top} \hat{a}_{\mathfrak{q}^{M}(t, \mathfrak{f}, \mathbf{w})}\left(\mathbf{e}_{i}\right) \mathbf{e}_{i}\right) d t,
\end{aligned}
$$

where the first term on the left is an Itô differential. Second, by (5.2),

$$
\begin{gathered}
-\sum_{i=1}^{n} \hat{\Pi}^{0} \hat{O}(t, \mathfrak{f}, \mathbf{w})^{\top} \hat{a}_{\mathfrak{q}^{M}(t, \mathfrak{f}, \mathbf{w})}\left(\mathbf{e}_{i}\right) \mathbf{e}_{i} \\
=\sum_{i=1}^{n} \hat{O}(t, \mathfrak{f}, \mathbf{w})^{\top} \hat{a}_{\mathfrak{q}^{M}(t, \mathfrak{f}, \mathbf{w})}\left(\mathbf{e}_{i}\right) \hat{\Pi}_{\mathfrak{q}^{M}(t, \mathfrak{f}, \mathbf{w})}^{\perp} \mathbf{e}_{i} .
\end{gathered}
$$

Finally, because, for each $t$ and $\mathbf{w}$, the expressions in the preceding are independent of the choice of the orthonormal basis $\left(\mathbf{e}_{1}, \ldots, \mathbf{e}_{n}\right)$, by choosing the basis so that each element is either orthogonal to or in $\mathfrak{q}^{M}(t, \mathfrak{f}, \mathbf{w})^{-1} T_{q^{M}(t, \mathfrak{f}, \mathbf{w})} M$, we see that the right hand side must vanishes identically. In other words,

$$
\hat{\Pi}^{0} \mathbf{W}=\hat{\Pi}^{0} \tilde{\mathbf{w}} \quad \text { when } \tilde{\mathbf{w}}(t) \equiv \int_{0}^{t} \hat{O}(\tau, \mathfrak{f}, \mathbf{w}) d \mathbf{w}(\tau) .
$$

Because $\hat{O}(\cdot, \mathfrak{f}, \mathbf{w})$ takes its values in $\mathcal{O}\left(\mathbb{R}^{n}\right)$ and the preceding integral is taken in the sense of Itô, $\tilde{\mathbf{w}}$ is an $\mathbb{R}^{n}$-valued Wiener process.

Remark: It is amusing to recognize that the difference between $\tilde{\mathbf{w}}(t)$ and $\mathbf{W}(t)$ is precisely

$$
\begin{gathered}
\frac{1}{2} \int_{0}^{t} \hat{O}(\tau, \mathfrak{f}, \mathbf{w})^{\top} \mathfrak{q}^{M}(\tau, \mathfrak{f}, \mathbf{w})^{-1} B_{q^{M}(\tau, \mathfrak{f}, \mathbf{w})} d \tau \\
=\frac{1}{2} \int_{0}^{t} \mathfrak{r}(\tau, \mathfrak{f}, \mathbf{w})^{-1} B_{q^{M}(\tau, \mathfrak{f}, \mathbf{w})} d \tau
\end{gathered}
$$

where (cf. (1.11)) B is the mean curvature vector.

\section{A Technical Addendum about Cartan's Structural Equations and Gauss's Formula.}

Use $\mathcal{O}(M)$ to denote the bundle of orthonormal frames over $M$, and define the solder form $\phi$ and connection 1-form $\omega$ accordingly (cf. page 181 of 
[3]). Next, let $\left(\mathbf{e}_{1}, \ldots, \mathbf{e}_{n}\right)$ be an orthonormal basis in $\mathbb{R}^{n}$ with $\mathbf{e}_{i} \in \mathbb{R}^{m}$ for $1 \leq i \leq m$. It should be apparent that the map $F: \mathcal{O}^{N}(M) \longrightarrow$ $\mathcal{O}(M)$ which takes $\mathfrak{f} \in \mathcal{O}^{N}(M)$ into $\left(\pi \mathfrak{f},\left(\mathfrak{f} \mathbf{e}_{1}, \ldots, \mathfrak{f} \mathbf{e}_{m}\right)\right) \in \mathcal{O}(M)$ is a smooth surjection which preserves the bundle structure. In addition, one sees that $\phi^{M} \equiv \phi\left\lceil\mathcal{O}^{N}(M)\right.$ and $\omega^{M} \equiv \hat{\Pi}^{0} \circ \omega \circ \hat{\Pi}$ are, respectively, the pullbacks under $F$ of the solder form $\phi$ and connection 1-form $\omega$ on $\mathcal{O}(M)$. Similarly, for each $\boldsymbol{\xi} \in \mathbb{R}^{m}, F_{*} \mathfrak{E}^{M}(\boldsymbol{\xi})_{\mathfrak{f}}$ is the horizontal lift to $F(\mathfrak{f}) \in \mathcal{O}(M)$ of $F(\mathfrak{f}) \boldsymbol{\xi} \in$ $T_{\pi \mathfrak{f}} M$. In other words, $F_{*} \mathfrak{E}^{M}(\boldsymbol{\xi})$ is not only well-defined, it is the canonical horizontal vector field on $\mathcal{O}(M)$ corresponding to $\left(\xi_{1}, \ldots, \xi_{m}\right)$. In particular, $-\omega\left(F_{*}\left[\mathfrak{E}^{M}(\boldsymbol{\xi}), \mathfrak{E}^{M}(\boldsymbol{\eta})\right]\right)$ is the curvature 2-form (cf. (8.44) in [3]) on $\mathcal{O}(M)$ at $F(\mathfrak{f})$.

All the above considerations should make one suspect that $\phi^{M}$ and $\omega^{M}$ might satisfy the Cartan Structural equations (cf. page 194 in [3]), and that the computation of $\left[\mathfrak{E}^{M}(\boldsymbol{\xi}), \mathfrak{E}^{M}(\boldsymbol{\eta})\right]$ ought to lead to an interesting form of Gauss's formula (cf. (3.27) in [2]). The key to verifying these suspicions is contained in the following lemma.

Lemma 6.1. There is a map

$$
\mathfrak{f} \in \mathcal{O}^{N}(M) \longmapsto \Omega_{\mathfrak{f}}^{M} \in \operatorname{Hom}\left(\mathbb{R}^{m} \times \mathbb{R}^{m} ; o^{\mathbb{R}^{n}}\left(\mathbb{R}^{m}\right)\right)
$$

such that, for each $(\boldsymbol{\xi}, \boldsymbol{\eta}) \in \mathbb{R}^{m} \times \mathbb{R}^{m}$

$$
\left[\mathfrak{E}^{M}(\boldsymbol{\xi}), \mathfrak{E}^{M}(\boldsymbol{\eta})\right]_{\mathfrak{f}}=-\lambda\left(\Omega_{\mathfrak{f}}^{M}(\boldsymbol{\xi}, \boldsymbol{\eta})\right)_{\mathfrak{f}}, \quad \mathfrak{f} \in \mathcal{O}^{N}(M) .
$$

Moreover, for each $\boldsymbol{\xi} \in \mathbb{R}^{m}$ and $a \in o\left(\mathbb{R}^{n}\right)$,

$$
\lambda(a)_{\mathfrak{f}} \hat{a}(\boldsymbol{\xi})=\left[\hat{a}_{\mathfrak{f}}(\boldsymbol{\xi}), a\right]+\hat{a}_{\mathfrak{f}}(a \boldsymbol{\xi}), \quad \mathfrak{f} \in \mathcal{O}^{N}(M)
$$

Finally, if $\boldsymbol{\xi} \in \mathbb{R}^{m}$ and $a \in o^{\mathbb{R}^{n}}\left(\mathbb{R}^{m}\right)$, then

$$
\left[\lambda(a), \mathfrak{E}^{M}(\boldsymbol{\xi})\right]_{\mathfrak{f}}=\mathfrak{E}^{M}(a \boldsymbol{\xi})_{\mathfrak{f}} .
$$

Proof. Proving the existence of $\Omega^{M}$ with the required properties is equivalent to checking that $\left[\mathfrak{E}^{M}(\boldsymbol{\xi}), \mathfrak{E}^{M}(\boldsymbol{\eta})\right]_{\mathfrak{f}}$ is vertical. But, by $(4.2)$, we know that

$$
\mathfrak{E}^{M}(\boldsymbol{\xi})_{\mathfrak{f}} \circ \mathfrak{E}^{M}(\boldsymbol{\eta})(\varphi \circ \pi)=\left\langle\mathfrak{f} \boldsymbol{\xi}, \operatorname{hess}_{\pi \mathfrak{f}}^{M} \varphi \mathfrak{f} \boldsymbol{\eta}\right\rangle,
$$

which, because the connection on $M$ is Levi-Civita's, means that $\pi_{*}\left[\mathfrak{E}^{M}(\boldsymbol{\xi}), \mathfrak{E}^{M}(\boldsymbol{\eta})\right]_{\mathfrak{f}}=0$. 
To check (6.3), simply note that

$$
\lambda(a)_{\mathfrak{f}} \hat{a}(\boldsymbol{\xi})=\left.\frac{d}{d s} e^{-s a} \hat{a}_{\mathfrak{f}}\left(e^{s a} \boldsymbol{\xi}\right)_{\mathfrak{f}} e^{s a}\right|_{s=0}=\left[\hat{a}_{\mathfrak{f}}(\boldsymbol{\xi}), a\right]+\hat{a}_{\mathfrak{f}}(a \boldsymbol{\xi}) .
$$

To prove (6.4), let $a \in o^{\mathbb{R}^{n}}\left(\mathbb{R}^{m}\right)$ be given, and use (8.5) and (8.15) in [3] together with (6.2) above to justify

$$
\begin{aligned}
& {\left[\lambda(a), \mathfrak{E}^{M}(\boldsymbol{\xi})\right]_{\mathfrak{f}}=\mathfrak{E}(a \boldsymbol{\xi})_{\mathfrak{f}}+[\lambda(a), \lambda(\hat{a}(\boldsymbol{\xi}))]_{\mathfrak{f}}} \\
& =\mathfrak{E}^{M}(a \boldsymbol{\xi})_{\mathfrak{f}}+\lambda\left(\left[a, \hat{a}_{\mathfrak{f}}(\boldsymbol{\xi})\right]+\lambda(a) \hat{a}(\boldsymbol{\xi})-\hat{a}_{\mathfrak{f}}(a \boldsymbol{\xi})\right)_{\mathfrak{f}}=\mathfrak{E}^{M}(a \boldsymbol{\xi})_{\mathfrak{f}} .
\end{aligned}
$$

To see that Cartan's structural equations hold for $\phi^{M}$ and $\omega^{M}$, one can now use exactly the same procedure as was used on page 194 of [3]. That is, one calculates $d \phi^{M}(\mathfrak{X}, \mathfrak{Y})$ and $d \omega^{M}(\mathfrak{X}, \mathfrak{Y})$ at $\mathfrak{f} \in \mathcal{O}^{N}(M)$ by considering what happens when $\mathfrak{X}$ and $\mathfrak{Y}$ are either $\mathfrak{E}^{M}(\boldsymbol{\xi})$, for some $\boldsymbol{\xi} \in \mathbb{R}^{m}$. or $\lambda(a)$, for some $a \in o^{\mathbb{R}^{n}}\left(\mathbb{R}^{m}\right)$. By using (8.5) in [3] together with (6.2) and (6.4) above, these computations lead immediately to the Cartan structural equations:

$$
d \phi^{M}=-\omega^{M} \wedge \phi^{M} \text { and } \quad d \omega^{M}=\omega^{M} \wedge \omega^{M}+\Omega^{M} \circ \phi^{M},
$$

where $\Omega^{M} \circ \phi^{M}(\mathfrak{X}, \mathfrak{Y}) \equiv \Omega^{M}\left(\phi^{M}(\mathfrak{X}), \phi^{M}(\mathfrak{Y})\right)$.

Finally, we want to find an expression for $\Omega^{M}$ in terms of the curvature 2 -form $\Omega$ for $\mathcal{O}(N)$. To this end, observe that

$$
\begin{aligned}
{\left[\mathfrak{E}^{M}(\boldsymbol{\xi}), \mathfrak{E}^{M}(\boldsymbol{\eta})\right]=[\mathfrak{E}(\boldsymbol{\xi}), \mathfrak{E}(\boldsymbol{\eta})]+[\mathfrak{E}(\boldsymbol{\xi}), \lambda(\hat{a}(\boldsymbol{\eta}))] } \\
\\
+[\lambda(\hat{a}(\boldsymbol{\xi})), \mathfrak{E}(\boldsymbol{\eta})]+[\lambda(\hat{a}(\boldsymbol{\xi})), \lambda(\hat{a}(\boldsymbol{\eta}))] .
\end{aligned}
$$

By definition,

$$
[\mathfrak{E}(\boldsymbol{\xi}), \mathfrak{E}(\boldsymbol{\eta})]=-\lambda(\Omega(\boldsymbol{\xi}, \boldsymbol{\eta}))
$$

By $(8.5)$ in [3],

$$
\begin{gathered}
{[\mathfrak{E}(\boldsymbol{\xi}), \lambda(\hat{a}(\boldsymbol{\eta}))]+[\lambda(\hat{a}(\boldsymbol{\xi})), \mathfrak{E}(\boldsymbol{\eta})]} \\
=\mathfrak{E}(\hat{a}(\boldsymbol{\eta}) \boldsymbol{\xi}-\hat{a}(\boldsymbol{\xi}) \boldsymbol{\eta})+\lambda(\mathfrak{E}(\boldsymbol{\xi}) \hat{a}(\boldsymbol{\eta})-\mathfrak{E}(\boldsymbol{\eta}) \boldsymbol{\xi}) .
\end{gathered}
$$

Notice that, because $\mathfrak{f} \boldsymbol{\xi}, \mathfrak{f} \boldsymbol{\eta} \in T_{\pi \mathfrak{f}} M,(1.6)$ implies that

$$
\hat{a}_{\mathfrak{f}}(\boldsymbol{\xi}) \boldsymbol{\eta}-\hat{a}_{\mathfrak{f}}(\boldsymbol{\eta}) \boldsymbol{\xi}=\mathfrak{f}^{-1}(\mathcal{S}(\mathfrak{f} \boldsymbol{\xi}) \mathfrak{f} \boldsymbol{\eta}-\mathcal{S}(\mathfrak{f} \boldsymbol{\eta}) \mathfrak{f} \boldsymbol{\xi})=0 .
$$


At the same time, by (2.2),

$$
\begin{aligned}
\mathfrak{E}(\boldsymbol{\xi}) \hat{a}(\boldsymbol{\eta})-\mathfrak{E}(\boldsymbol{\eta}) \hat{a}(\boldsymbol{\xi}) & =([\mathfrak{E}(\boldsymbol{\xi}), \mathfrak{E}(\boldsymbol{\eta})] \hat{\Pi}) \circ\left(\hat{\Pi}-\hat{\Pi}^{\perp}\right)+2[\hat{\mathcal{S}}(\boldsymbol{\eta}), \hat{\mathcal{S}}(\boldsymbol{\xi})] \\
& =-[\hat{\Pi}, \Omega(\boldsymbol{\xi}, \boldsymbol{\eta})] \circ\left(\hat{\Pi}-\hat{\Pi}^{\perp}\right)-2[\hat{\mathcal{S}}(\boldsymbol{\xi}), \hat{\mathcal{S}}(\boldsymbol{\eta})] .
\end{aligned}
$$

Thus,

$$
\begin{aligned}
{[\mathfrak{E}(\boldsymbol{\xi}), \lambda(\hat{a}(\boldsymbol{\eta}))] } & +[\lambda(\hat{a}(\boldsymbol{\xi})), \mathfrak{E}(\boldsymbol{\eta})] \\
& =-\lambda\left(\left[\hat{\Pi}, \Omega(\boldsymbol{\xi}, \boldsymbol{\eta}) \circ\left(\hat{\Pi}-\hat{\Pi}^{\perp}\right)\right]+2[\hat{\mathcal{S}}(\boldsymbol{\xi}), \hat{\mathcal{S}}(\boldsymbol{\eta})]\right) .
\end{aligned}
$$

Finally, by (6.3),

$$
\begin{aligned}
{[\lambda(\hat{a}(\boldsymbol{\xi})), \lambda(\boldsymbol{\eta})] } & =\lambda([\hat{a}(\boldsymbol{\xi}), \hat{a}(\boldsymbol{\eta})]+\lambda(\hat{a}(\boldsymbol{\xi})) \hat{a}(\boldsymbol{\eta})-\lambda(\hat{a}(\boldsymbol{\eta})) \hat{a}(\boldsymbol{\xi})) \\
& =-\lambda([\hat{a}(\boldsymbol{\xi}), \hat{a}(\boldsymbol{\eta})])=\lambda([\hat{\mathcal{S}}(\boldsymbol{\xi}), \hat{\mathcal{S}}(\boldsymbol{\eta})]) .
\end{aligned}
$$

Hence, when we put this together with (a) and (b) and plug them into $\left(^{*}\right)$, we conclude that

$$
\begin{aligned}
& {\left[\mathfrak{E}^{M}(\boldsymbol{\xi}), \mathfrak{E}^{M}(\boldsymbol{\eta})\right]} \\
& \quad=-\lambda\left(\Omega(\boldsymbol{\xi}, \boldsymbol{\eta})+[\hat{\Pi}, \Omega(\boldsymbol{\xi}, \boldsymbol{\eta})] \circ\left(\hat{\Pi}-\hat{\Pi}^{\perp}\right)+[\hat{\mathcal{S}}(\boldsymbol{\xi}), \hat{\mathcal{S}}(\boldsymbol{\eta})]\right) \\
& \quad=-\lambda\left(\hat{\Pi} \circ \Omega(\boldsymbol{\xi}, \boldsymbol{\eta}) \circ \hat{\Pi}+\hat{\Pi}^{\perp} \circ \Omega(\boldsymbol{\xi}, \boldsymbol{\eta}) \circ \hat{\Pi}^{\perp}+[\hat{\mathcal{S}}(\boldsymbol{\xi}), \hat{\mathcal{S}}(\boldsymbol{\eta})]\right) .
\end{aligned}
$$

This not only give another proof that $\left[\mathfrak{E}^{M}(\boldsymbol{\xi}), \mathfrak{E}^{M}(\boldsymbol{\eta})\right]$ is vertical, it shows that

$$
\Omega^{M}(\boldsymbol{\xi}, \boldsymbol{\eta})=\hat{\Pi} \circ \Omega(\boldsymbol{\xi}, \boldsymbol{\eta}) \circ \hat{\Pi}+\hat{\Pi}^{\perp} \circ \Omega(\boldsymbol{\xi}, \boldsymbol{\eta}) \circ \hat{\Pi}^{\perp}+[\hat{\mathcal{S}}(\boldsymbol{\xi}), \hat{\mathcal{S}}(\boldsymbol{\eta})] .
$$

To see that (6.6) gives the Gauss formula relating the Riemann curvature $R^{M}$ on $M$ to the Riemann curvature $R^{N}$ on $N$, recall (cf. (8.54) in [3]) that the Cartan structural equations lead to

$$
R^{N}\left(X_{\pi \mathfrak{f}}, Y_{\pi \mathfrak{f}}\right)=\mathfrak{f} \circ \Omega\left(\mathfrak{f}^{-1} X_{\pi \mathfrak{f}}, \mathfrak{f}^{-1} Y_{\pi \mathfrak{f}}\right) \circ \mathfrak{f}^{-1}
$$

for all $\mathfrak{f} \in \mathcal{O}(N)$ and $X_{\pi \mathfrak{f}}, Y_{\pi \mathfrak{f}} \in T_{\pi \mathfrak{f}} N$. In the same way, (6.5) shows that

$$
R^{M}\left(X_{\pi \mathfrak{f}}, Y_{\pi \mathfrak{f}}\right)=\mathfrak{f} \circ \hat{\Pi}_{\mathfrak{f}} \circ \Omega^{M}\left(\mathfrak{f}^{-1} X_{\pi \mathfrak{f}}, \mathfrak{f}^{-1} Y_{\pi \mathfrak{f}}\right) \circ \hat{\Pi}_{\mathfrak{f}} \circ \mathfrak{f}^{-1}
$$

for all $\mathfrak{f} \in \mathcal{O}^{N}(M)$ and $X_{\pi \mathfrak{f}}, Y_{\pi \mathfrak{f}} \in T_{\pi \mathfrak{f}} M$. Hence, (6.6) leads to Gauss's formula

$$
R^{M}\left(X_{x}, Y_{x}\right)=\Pi_{x} \circ\left(R^{N}\left(X_{x}, Y_{x}\right)+\left[\mathcal{S}_{x}\left(X_{x}\right), \mathcal{S}\left(Y_{x}\right)\right]\right)\left\lceil T_{x} M\right.
$$

for all $x \in M$ and $X_{x}, Y_{x} \in T_{x} M$. 


\section{References.}

[1] Bishop, R. \& Crittenden, R., Geometry of Manifolds, Pure \& Appl. Math. Series \#15, Academic Press, 1964.

[2] Sakai, T., Riemannian Geometry, Translations of Mathematical Monographs, vol. 149, AMS, Providence, RI, 1996.

[3] Stroock, D., An Introduction to the Analysis of Paths on a Riemannian Manifold, Mathematical Surveys and Monographs, vol. 74, AMS, Providence, RI, 1999.

M.I.T., 2-272, CAMBridge, MA 02139-4307, USA

dws@math.mit.edu 